Estudios Románicos, Volumen 27, 2018, pp. 225-236

ISSN: 0210-491

eISSN: 1989-614X

DOI: https://doi.org/10.6018/ER/346661

\title{
LE SOURIRE : CONCEPTION VS PERCEPTION (SUR L'EXEMPLE DE LA COMMUNICATION NON VERBALE DES FRANÇAIS, DES ESPAGNOLS ET DES RUSSES)
}

(Smile in French, Spanish and Russian nonverbal communication: conception $v s$ perception)

\author{
Elena Sandakova* \\ Université d'Alicante
}

\begin{abstract}
The present study is placed within the context of kinesics and emphasises the importance of facial and corporal gestures in this kind of communication in foreign languages, taking into consideration the fact that all interactants are social actors in a multicultural reality. The analysis is based on the considerations of such authors as R. Birdwhistell, A. \& B. Pease, P. Brown, S. Levinson, and C. Kerbrat-Orecchioni among others. Specifically, it is focused on the conception of smile as a part of nonverbal politeness and visual language (photos) in settings of French, Spanish and Russian languages and cultures, as well as with the perception of smile among speakers of the communities under examination. Finally, the results of this work show that there is a disequilibrium between the perception of smile by out-group and its conception within in-group that is explained by ignorance of the social norms prevailing in nonverbal politness of the target culture, as well as by the persistance of stereotypes.
\end{abstract}

Key words: Kinesics; Gesture; Nonverbal politeness; Smile; Photo; Stereotype.

Résumé : L'étude présente se place dans le domaine de la kinésique et met en relief l'importance de connaître les gestes faciaux et corporels en langue étrangère, d'autant plus que nous sommes tous des acteurs sociaux d'une réalité pluriculturelle. L'article prend appui sur les considérations de R. Birdwhistell, A. et B. Pease, P. Brown, S. Levinson, C. Kerbrat-Orecchioni, et se concentre généralement sur le traitement de la concep-

* Adresse pour la correspondance : Elena Sandakova. Departamento de Filologías Integradas. Facultad de Filosofía y Letras. Universidad de Alicante. Ctra. San Vicente del Raspeig, s/n, 03690 San Vicente del Raspeig, Alicante (elena.sand@ua.es) 
tion du sourire dans le cadre de la politesse non verbale et du langage visuel (photos) dans les espaces linguistico-culturels français, espagnol et russe, aussi bien que de sa perception parmi les usagers des communautés en question. Les résultats de l'analyse montrent qu'il y a un déséquilibre entre la perception du sourire par l'exogroupe et sa conception dans l'endogroupe qui est expliqué par la méconnaissance des normes sociales régnant dans la politesse non verbale de la nation cible, aussi bien que par la persistance des stéréotypes.

Mots clés : Kinésique ; Geste ; Politesse non verbale ; Sourire ; Photographie ; Stéréotype.

\section{Introduction}

Avec une compétence de communication désormais plurilingue et pluriculturelle, on sait d'emblée que la communication non verbale est nécessaire pour maîtriser les échanges avec les étrangers.

Le langage non verbal, ou tout le répertoire de la gestualité, s'inclut dans le cadre de la science « kinésique », initiée par l'anthropologue américain Ray Birdwhistell dans les années cinquante du siècle dernier. Conçue en tant qu'étude des mouvements faciaux et corporels, elle s'avérait être un homologue de la phonologie : « the kinesic system has forms which are astonishingly like words in language » (Birdwhistell 1970:80). Le geste, en tant qu'objet de la kinésique, est repris chez les chercheurs postérieurs et désigne une expression du visage, une pose, un mouvement des mains, des pieds, de la tête et une manière du corps (Krejdlin $2008: 7$ ).

Au sein de la gestuelle, notre intérêt est spécialement porté sur le mouvement facial le plus universel qui est le sourire. Avec cet objet de l'étude, nous mettons en relation les sujets de la recherche (les usagers des espaces linguistico-culturels français, espagnol et russe) avec le but de comparer la conception du sourire dans ces cultures, ainsi que de la contraster avec la perception du geste par les étrangers. Le premier essai à ce sujet a été entrepris par nous il y a quelques années en forme d'un article adressé aux Russes pour les préparer à une interaction plus symétrique avec les Français et les Espagnols (Sandakova 2011). L'étude présente est donc un vecteur au mouvement inverse dont le but est de compléter le panorama pluriculturel englobant les communautés en question.

Les observations empiriques de la réception du sourire, recueillies par nous les dernières années, dans l'endogroupe, i.e. groupe interne, et les exogroupes, i.e. autres groupes, anticipent que la méconnaissance de la politesse non verbale des étrangers consolide les hétéro-stéréotypes (i.e. croyances et représentations sur un exogroupe) déjà ancrés et éloigne progressivement du démenti des préjugés. Ces observations se basent sur un corpus de :

- témoignages oraux et écrits de parleurs et d'écrivains (professeurs, acteurs, entre autres) publiés dans des livres ( $C f$. V. Berkov et al., A. Sergueyeva, A. Perevoznikova, T. Yegorova, N. Volskaya, D. Lapierre), blogs d'Internet, forums, aussi bien que dans les programmes de télévision (Callejeros Viajeros, Españoles en el 
mundo). Les sources consultées se sont avérées essentielles pour notre recherche, bien qu'il soit à reconnaître leur nombre divers et abondant ;

- photos sur les réseaux sociaux et les applications mobiles (Facebook, Instagram, Vkontakte, Odnoklassniki, WhatsApp, Telegram). Étant d'accès public libre, elles ont été obtenues à partir des profils et des albums électroniques. Tout de même, au cours de l'article, nous maintiendrons le caractère anonyme des Spectrums et des Operators. Nous nous abstiendrons de même de publier les photos postées, étant donné que révéler la personnalité ne constitue pas notre objectif.

Une hypothèse générale et l'autre, spéciale, en découlent : Pour accéder au vrai sens de la culture cible et mieux comprendre ses porteurs, le puisement dans les signes non verbaux est-il plus efficace que les idées préconçues et généralisées ? La perception du sourire par l'exogroupe aboutit-elle à ébaucher un masque correct de l'endogroupe ?

Les réponses à ces questions réclament, en premier lieu, une révision théorique du sourire comme phénomène important du langage non verbal, du lieu de ce geste dans les typologies des chercheurs, de son choix dans la politesse non verbale et de sa manifestation selon les normes internes en vigueur s'étendant même sur la photographie.

\section{Sourire comme élément de la kinésique}

Le sourire comme mouvement facial universel au sein du classement de la gestuelle nous renvoie, avant tout, à Allan et à Barbara Pease qui ont défendu le statut des gestes universels : « les différences culturelles sont nombreuses, mais les signes basiques du langage du corps sont les mêmes partout $»^{1}$ (Pease 2006 : 34). Le couple y rapporte des mouvements innés et inconscients, contrairement aux gestes qui s'acquièrent consciemment lors de la communication. Les gestes universels se basent ainsi sur des raisons purement physiologiques : par exemple, le geste de mouvoir la tête d'un côté à l'autre indique «non » et doit son origine à l'alimentation dans l'enfance (Pease 2006 : 34) ; le fait moderne de se moucher est le résultat des anciennes épidémies de tuberculose (Pease 2006 : 134). Ajoutons-y le geste de hausser les épaules qui veut dire vouloir protéger le cou des objets menaçants possibles et qui exprime la subordination devant la situation où on ne comprend ou on ne sait rien. Enfin, c'est le geste « cœur ", formé à l'aide des index et des pouces des deux mains, esquissant les contours du cœur comme organe du corps humain, qui implique l'amour.

En tant que geste universel, le sourire provient également d'une raison primitive : «Le sourire est un signe de soumission $»^{2}$ (Pease $2006: 84$ ). Chez les humains, le sourire communique que le destinataire n'est pas une menace pour le locuteur et, en plus, ce dernier demande de l'accepter au niveau personnel. Dans l'occurrence, A. et B. Pease recourent à l'exemple du président de la Fédération de Russie V. Poutine, dont l'aspect peu souriant les fait conclure qu'il refuse de paraître soumis.

1 Traduit par nos soins.

2 Traduit par nos soins. 
Au sein de la typologie de la gestuelle de David Efron, entreprise par Harold Johnson, Paul Ekman et Wallace Friesen, le sourire fait partie des gestes adaptateurs. Ceuxci sont des mouvements spontanés des émotions réalisés afin de donner du confort à l'individu lors de l'interaction. Outre le sourire, les gestes les plus représentatifs qui peuvent adapter les humains lors de la communication sont passer la main dans les cheveux, se gratter la tête, retirer un cheveu sur son vêtement, croiser (décroiser) les jambes, s'étirer, froncer les sourcils, baisser la tête, etc. (Johnson, Ekman, Friesen 1975 : 335).

Paola Bertocchini et Edvige Costanzo entreprennent leur classification des gestes d'après les fonctions communicatives. Le sourire s'y trouve parmi les gestes à fonction proprement communicative dont le but est de synchroniser l'action. Cependant, le sourire n'est pas phatique comme le contact par le regard, ni régulateur comme des hochements de tête ; il n'est qu'un rite d'interaction tout comme le salut ou la courbette (Bertocchini, Costanzo 2008 : 54-55).

Le sourire s'inscrit finalement dans le panorama des valeurs fonctionnelles des gestes établi par le linguiste russe Grigorij Krejdlin. Les gestes peuvent répéter ou doubler une information, remplacer des propos oraux, souligner ou renforcer un élément du discours, compléter le contenu du discours, régulariser la communication et voire contredire le signifié parlé. En particulier, le sourire peut contredire les énoncés « Je suis très mal » et « J'ai échoué à l'examen » (Krejdlin 2008 : 10-11).

\section{Sourire comme stratégie de la politesse non verbale}

La politesse est une catégorie communicative universelle composée de stratégies comportementales qui déterminent le choix des unités de la communication verbale et non verbale. Outre divers modes d'adresses verbales courtoises, on peut observer ceux d'échanges gestuels, mimiques et tactiles, tels qu'embrassades, poignées de main, accolades, regards, sourires, etc.

Depuis la démarche pluriculturelle, la notion «politesse », en tant que manifestation du respect et de l'attention, ne s'avère pas être adéquate. Étant donné que les concepts « respect » et « attention » s'interprètent différemment d'une culture à l'autre, la notion « politesse » ne revêt donc pas de signifié universel ni partagé par tous.

Le premier élan à l'étude de la politesse comme catégorie de la conscience communicative a été donné par Penelope Brown et Stephen Levinson. Leur modèle de politesse se fonde sur la considération de l'individu en tant qu'entité sociale et sur la distinction qui en résulte entre (i) la protection du territoire du soi et (ii) la valorisation de l'image de l'allocutaire. Cette distinction de base a reçu les noms, consécutivement, de " politesse négative » et de « politesse positive » (Brown, Levinson 1987 : 60).

Dans l'occurrence, les chercheurs anglais s'inspirent de la « théorie des faces » développée dans les années soixante-dix par Erving Goffman. La face est définie par ce dernier comme valeur positive sociale demandée par le participant à une conversation (Goffman 1972 : 319). Postérieurement, P. Brown et S. Levinson la reprennent comme entité des deux désirs de base que tout être peut présenter et qui constituent un aspect fondamental de son rapport au monde (Brown, Levinson 1987 : 61-62). De la sorte, la politesse positive dérive 
de la face positive et correspond à la façade sociale, à l'amour-propre, au narcissisme, à l'image valorisante de l'individu qu'il s'efforce de présenter à l'extérieur. Elle se trouve donc au cœur du comportement familier et de la plaisanterie ; elle traduit le désir de l'individu à se rapprocher, à être suivi et apprécié des autres. En revanche, la politesse négative s'adresse à la face négative de l'individu et traduit son désir à se maintenir distant et à ne pas être dérangé. Elle correspond donc au territoire de chacun (le corps, l'intimité, la vie privée, etc.) et est situé au cœur du comportement de respect (Brown, Levinson 1987 : 101, 129).

Les deux désirs de base, ceux de préserver les faces, sont protégés par l'individu car, pour l'une comme pour l'autre, il existe constamment des menaces potentielles. P. Brown et S. Levinson les appellent « Face Threatening Acts» (actes menaçants pour la face) et les abrègent en FTA (Brown, Levinson 1987 : 60). Ainsi, selon la face menacée de chacun des interlocuteurs, ils répartissent les actes de langage en quatre catégories :

1. Des FTA(s) pour la face positive du locuteur : par exemple, l'autocritique.

2. Des FTA(s) pour la face négative du locuteur : les offres et les promesses que le locuteur fait à ses dépens, concernant le sacrifice de son temps, de ses forces, de ses intérêts, de son énergie.

3. Des FTA(s) pour la face positive de l'allocutaire : les insultes, les injures, les moqueries, la critique, etc.

4. Des FTA(s) pour la face négative de l'allocutaire : les ordres, les requêtes, les instructions, les questions indiscrètes, etc.

Le modèle de politesse de $\mathrm{P}$. Brown et $\mathrm{S}$. Levinson, portant un caractère abstrait, tend à ménager et à valoriser les faces de l'autre sans faire trop de mal aux siennes propres, ou, en d'autres mots, à instaurer, selon Roland Barthes « un état d'équilibre très subtil et très fin pour se protéger sans blesser l'autre » (Barthes 2000). La conciliation des intérêts entre les locuteurs, selon ce dernier, est presqu'acrobatique.

Catherine Kerbrat-Orecchioni remanie le modèle de politesse de P. Brown et S. Levinson en la plaçant en contexte. Plus particulièrement, c'est la situation communicative qui détermine si un énoncé est poli et approprié, ou impoli et inapproprié. L'auteure renvoie la politesse positive au caractère interventionniste et la politesse négative, au caractère abstentionniste (Kerbrat-Orecchioni 1992 : 177). La première consiste à accomplir un acte intrinsèquement poli et à valoriser un FTA, tandis que la dernière consiste à éviter un FTA ou à l'adoucir.

Le modèle de politesse de C. Kerbrat-Orecchioni devient donc équilibré par le concept d'« anti-FTA » ou FFA (Face Flattering Acts), c'est-à-dire « actes valorisants pour la face ». Chaque acte de langage peut s'avérer un FTA, un FFA ou l'ensemble des deux. Sur son exemple d'un joueur de football qui se flatte devant l'intervieweur, la linguiste illustre que son acte est un FFA par rapport à sa face, au même temps qu'il s'agit d'un FTA par rapport aux faces positives et négatives de l'interlocuteur, puisque ce dernier se voit brusqué devant une telle mégalomanie (Kerbrat-Orecchioni 2001 : 74).

Dans un tel cadre, entre la catégorie de politesse positive et celle de politesse négative, C. Kerbrat-Orecchioni ressent la nécessité d'en introduire d'autres. Selon la confor- 
mité de l'énoncé aux normes en vigueur dans une situation communicative donnée, elle définit les catégories suivantes :

- Politesse - présence de marqueurs plus ou moins conformes aux attentes normatives en vigueur de la situation. Elle est révélée d'après les marqueurs d'adoucissement (FTA) et de renforcement (FFA).

- Hyperpolitesse - présence de marqueurs de politesse excessives par rapport aux attentes. C'est le cas de trop de politesse qui peut tuer la politesse. Or, elle peut basculer dans l'impolitesse si elle prend une valeur ironique ou sarcastique.

- A-politesse ou non-politesse - absence normale de marqueurs de politesse.

- Impolitesse - absence anormale de marqueurs de politesse ou présence de marqueurs d'impolitesse.

- Polirudesse - présence complexe des marqueurs liés aux catégories antérieures. Elle peut englober des FTA camouflés en FFA, i.e. des énoncés polis qui, en réalité, sont menaçants pour la face de l'interlocuteur (Kerbrat-Orecchioni 2011 : 98-99).

La politesse est un phénomène social puisqu'elle manifeste l'attitude des individus à l'égard des normes en vigueur. De même qu'on peut décrire le rapport de la nation à la distance (la proxémique), au temps (la chronémique) et au contexte, on peut parler du rapport de la nation à la politesse. Par conséquent, à côté de la division des cultures en celles «à fort degré de contact 》 et celles «à faible degré de contact» (Hall 1973: 184-194), en «monochrones » et «polychrones » (Hall 1989: 15-32; Hall $1981: 17-24$ ), en celles «à un contexte haut » et celles «à un contexte bas » (Hall 1981:91-116), nous pourrions instaurer la répartition des cultures en « polies» et « non polies ».

Les cultures polies sont donc des sociétés où prédomine la politesse positive. Les marqueurs-modèles y circulant ont pour fonction de renforcer les FFA au niveau verbal (remerciement, excuse, compliment, etc.), socioculturel (invitation, visite, cadeaux, etc.) et non verbal (bises, sourire, embrassades, etc.). Les membres de ces sociétés attachent une importance relative à la protection de la face positive et à la soutenance du narcissisme.

Les sociétés où prédomine la politesse négative disposent de marqueurs visés à adoucir les FTA. Les individus de ces sociétés privilégient, avant tout, leur face négative et préservent le territoire corporel de même que temporel et cognitif.

À travers le prisme des concepts théoriques traités ut supra, nous nous disposons à illustrer les particularités de la réception du sourire dans les espaces linguistico-culturels français, espagnol et russe. Rappelons que depuis la perspective pluriculturelle nous croyons également nécessaire de comparer la perception du sourire par l'exogroupe et sa conception dans/ chez l'endogroupe, d'après les normes sociales en vigueur.

En ce qui concerne le rapport à la politesse, la société française et la société espagnole sont, dans une plus grande mesure, des cultures polies. La politesse positive prévaut parmi leurs usagers, ses marqueurs adoucissent les FTA et renforcent les FFA au niveau non verbal, y compris le sourire. Face à ce sourire " poli » bien intégré et 
(sur l'exemple de la communication non verbale des Français, des Espagnols et des Russes)

privilégié dans les endogroupes, son absence normale y est conçue généralement comme apolie ou non polie. Par ailleurs, une présence excessive du sourire risque d'apporter trop d'amadouement à l'interaction et de s'avérer alors hyperpolie.

Sous l'influence de telles normes sociales internes de la politesse positive et du sourire, les Français et les Espagnols ont l'habitude de prendre les Russes pour sérieux et antipathiques : «Ils sont rustres et peu souriants » (Anne 2012); « Souvent, les Français disent : ah, les Russes sont vraiment froids, froids comme de la glace » (Adrien, Blog Apprendre le russe) ; "Ils sont froids, c'est vrai. Même pour moi, ils sont super froids [...] C'est vrai qu'ils ne sont pas habitués à sourire » (Inga, Blog Apprendre le russe); « Ni una sonrisa, ni un gesto; sólo un inefable asombro » (Lapierre $2006: 50) ;$ « [...] encontraba extraño que camareros o vendedores no sonrieran nunca » (Salvador 2010).

À son tour, la communauté russe ne peut pas s'appeler « société à politesse positive » en matière du sourire. À la différence du contexte français et espagnol, le russe manque du marqueur « le sourire poli ». Dans la société russe on peut constater la tendance à la prédominance de la politesse négative, i.e. le désir à se maintenir, toujours par égard et respect, dans sa coquille, distant, non dérangé, seul sur son territoire intime. De la sorte, le sourire parmi les Russes porte un caractère abstentionniste et ne vise pas à renforcer les FFA, mais à adoucir les FTA ou à les éviter. La présence du sourire dans les situations quotidiennes qui ne l'exigent pas marque l'a-politesse dans l'endogroupe. Sous le poids de ces normes internes, les Russes cherchent toujours à comprendre le sens du sourire, conçu comme « poli » et/ ou «normal » chez les Français et les Espagnols. D'après les forums, ils terminent par l'interpréter comme « sourire sans le sens », étant pour eux un indice de moquerie, de folie, de défi, d'hypocrisie, voire une allusion de caractère sexuel : «Souvent, les Russes à qui l'on a adressé un sourire répondent : 'Je ne comprends pas ce qu'il y a de drôle !'» (Sternin 2013).

À notre avis, le sourire, comme norme sociale, peut possiblement être déterminée par le facteur historique : un peuple peut avoir subi plus de grands avatars que l'autre. On pourrait y ajouter le facteur climatique : le froid dans un pays est plus sévère que dans l'autre, le soleil y brille moins, etc. N'excluons pas non plus le facteur social : qualité de la vie, rapports humains, etc. En somme, ces facteurs font que les individus d'une nation sourient moins (ou plus) que ceux d'une autre.

Hormis, le facteur étymologique peut également avoir lieu. En français, le mot « sourire » dérive de l'ancien français surrire, provenu, à son tour, du latin subridere, composé de ridere, 'rire', et sub-, préfixe marquant l'atténuation. Le $D R A E$ espagnol relève dans le mot sonrisa les formants son-, 'sous', et risa, 'rire'. Dans la langue russe, le mot « sourire », улыбка, provient du substantif лоб, 'front', dont un des signifiés était 'crâne' en vieux slave. Aussi, dans la vieille culture russe, le sourire s'associait-il au rictus du crâne.

Les valeurs fonctionnelles du sourire sont aussi comparables parmi les nations. Ainsi, chez les Français et les Espagnols, le sourire est un mouvement facial qui donne du confort lors de l'interaction. Mais chez les Russes le sourire est conçu comme transmetteur des sentiments, dont les plus partagés sont la cordialité, la confiance et l'amabilité. Selon la politesse positive ancrée dans la nation russe, toute amabilité, disposition, cordialité, confiance et bienveillance ne se manifestent pas par le biais du sourire, mais 
moyennant d'autres moyens : paraverbaux (expressions des yeux), prosodiques (intonation) et, surtout, verbaux.

Le sourire, en tant que rite d'interaction synchronisant, chez les Français et les Espagnols, traduit essentiellement les bonnes manières. En revanche, il n'équivaut pas nécessairement à la politesse pour les Russes, puisque pour eux, être poli avec l'allocutaire ne signifie pas lui sourire. Une vendeuse ou une secrétaire non souriante n'y serait jamais traitée d'impolie ni d'incompétente dans son travail. Cette norme comportementale est, en plus, attachée à un autre phénomène social dit « syndrome du concierge ». Il est propre aux représentants des postes administratifs qui disposent d'un pouvoir minime dans leurs mains et abusent de leurs fonctions de gestion. Considérés par eux-mêmes " petits chefs », ils ont toujours un aspect peu souriant, indifférent et froid (Volskaya 2001 : 11 ; Berkov 2003 : 13).

Tout de même, si un Russe sourit durant l'interaction, il montre qu'il est vraiment enchanté, ravi, content ou de bonne humeur.

Comme le montrent les opinions des forums russes étudiés, les derniers temps, la tenue sérieuse et peu souriante des Russes constitue un vrai auto-stéréotype, i.e. croyance sur soi-même et son groupe. Afin de l'anéantir les Russes empruntent les normes comportementales européennes, modifient, quoiqu'indirectement, le vecteur de leur politesse et imposent émotionnellement « le sourire poli ». Celui-ci se fait sentir, particulièrement, dans le secteur tertiaire où le but de fidéliser les rapports avec le client est plus que primordial. Vu le caractère forcé du geste, les natifs le qualifient de « sourire de circonstance ».

\section{Le sourire et la photographie}

Étant un chaînon important du langage non verbal, le sourire l'est simultanément dans le langage visuel. Ce n'est pas hasard que dans la technique photographique de chaque culture il existe un mot provoquant le sourire. Sa prononciation prétend mettre en action les muscles latérales du visage capables de se connecter avec les commissures de la bouche et les orbicularis oculti, et d'étirer les yeux en arrière. D'habitude, ce mot, soit de nature mono- ou polysyllabique, comporte le son /i/ : ouistiti (en français), cheese (en anglais), cыp /'siR/ (en russe); l'exception y serait patata (en espagnol), bien qu'on y prononce aussi le mot Luis. Peu importe sa traduction, l'émission du mot provoque obligatoirement un sourire. Cependant, certains signalent son caractère faux et n'approuvent pas « un aspect peu sincère de la photo » (Pease $2006: 83$ ).

Le sourire manifesté ou non sur les images visuelles, ou photos, peut constituer une croyance sur le groupe aussi bien que renforcer une idée préconçue sur celui-ci.

Conformément à notre étude visuelle, l'aperçu des photos informelles nommées « amateur », où apparaissent les Français, les Espagnols et les Russes, témoignent d'une certaine différence. En terme du sourire, les Spectrums ${ }^{3}$ français et espagnols ne sortent pas si maussades que les Russes. L'explication réside dans la manifestation «normale » du

3 D'après la terminologie de R. Barthes, les sujets photographiés (Barthes 1999 : 38). 
(sur l'exemple de la communication non verbale des Français, des Espagnols et des Russes)

sourire chez les premiers et son abstentionnisme naturel chez les derniers. Selon la même conception d'origine, les Spectrums russes ne sourient même pas sur les fameux selfies. À ce propos, le chercheur de l'étude visuelle « Selfiecity », Lev Manovich, ajoute que les auteurs des selfies suivent le culte du cool, qui, à son tour, se manifeste chez les mannequins qui posent sans sourire et deviennent objet d'imitation, surtout chez les femmes.

On ne voit nulle allusion au sourire dans les conseils d'une blogueuse pour faire l'auto-captage des avatars : "N'oublie pas de bien ouvrir les yeux et de faire la bouche en bec de canard [...] Il vaut mieux se prendre en photo d'en haut et avoir un regard mystérieux de dessous les franges » (Olga 2010).

De même, sous l'influence des normes sociales toujours en vigueur, les Spectrums russes ne sourient pas sur les photos à des fins administratives. Selon le Service fédéral de migration de la Fédération de Russie, l'expression du visage de la personne photographiée doit être neutre, les lèvres fermées. La norme s'étend sur toute photo formelle qui figure dans le passeport, le permis de conduire et d'autres documents identifiants officiels.

L'air sérieux et triste des Spectrums russes sur les photos administratives les a menés à un autre auto-stéréotype. Celui-ci s'est exprimé dans une blague du monologuiste russe contemporain Pavel Volya : «En jetant un coup d'œil sur nos photos des passeports, on dirait que tout le pays est recherché ». L'autre blague a été répandue par la chaîne de radio nationale, leader en Russie, Русское радио [Radio Russe] : "Si tu ressembles à ta photo du passeport, il est temps de prendre les vacances ».

\section{Conclusion}

Le sourire est une composante parlante du langage non verbal et visuel, et sa perception par un exogroupe n'est parfois pas la même que sa conception dans l'endogroupe. Outre sa fonction de donner du confort à l'individu lors de l'interaction, celle que nous avons privilégiée est la manifestation d'un rite, d'après les normes sociales en vigueur. Sur l'exemple des cultures française, espagnole et russe, nous avons voulu souligner la différence en normes sociales qui y règnent, aussi bien que l'importance de les prendre en considération lors du jugement sur l'exogroupe. Ainsi, à l'intérieur de la communauté russe la perception du sourire découle de quelques normes : (i) les Russes ne sont pas une "nation à politesse positive », (ii) le geste de sourire y sert à adoucir les FTA et à les éviter, ainsi qu'il transmet plus les émotions que les bonnes manières, (iii) le marqueur « le sourire poli » n'y existe pas pour des raisons plutôt étymologiques, (iv) la présence du sourire dans les interactions quotidiennes qui ne l'exigent pas marque l'apolitesse. Nous tendons donc à croire que si l'étranger puisait dans ce signe non verbal, sa perception serait proche à la conception d'origine, il ne tomberait pas dans le piège des stéréotypes (dont « Les Russes sont froids et antipathiques ») et s'adapterait mieux à l'interaction avec les Russes. À l'inverse, en percevant le sourire chez les Français et les

4 Interview avec Lev Manovich. [http://www.konbini.com/fr/tendances-2/entretien-selfiecitybase-de-donnees-selfie/; 10/10/2015]. 
Espagnols comme un vrai marqueur de politesse conventionnel, un Russe ne tiendrait à y chercher de signifié ni de prémisses émotionnelles (« Mais pourquoi il sourit ?»).

Le sourire et la politesse non verbale sont des phénomènes universels et kinésiquement pertinents dans la démarche pluriculturelle. Sur leur exemple, la maîtrise des conventions de l'exogroupe arrondirait les angles et " polirait» les rouages de la machine conversationnelle en développant l'interaction dans les meilleures conditions.

\section{BIBLIOGRAPHIE}

ANNE (2012) : «Les Russes ». Blog Annouchka. [http://bit.ly/2BRfOQ1 ; 27/02/2018].

ADRIEN : « Les Russes sont froids ? Voici comment 'briser la glace'». Blog Apprendre le russe. [http://bit.ly/2GRBdrL ; 27/02/2018].

BARTHES, Roland (1999): La cámara lúcida. Nota sobre la fotografía, (trad. J. SalaSanahuja). Barcelona: Paidós Comunicación.

(2000) : « Les saveurs du savoir ». Entretiens. France Culture, [émission].

BERTOCCHINI, Paola ; COSTANZO, Edvige (2008) : Manuel de formation pratique pour le professeur de français langue étrangère. Paris : CLÉ International.

BIRDWHISTELL, Ray (1970): Kinesics and Context. Essays on Body Motion Communication. Philadelphia: University of Pennsylvania Press.

BROWN, Penelope ; LEVINSON, Stephen (1987) : Politeness: Some universals in language usage. Cambridge : Cambridge University Press.

Callejeros viajeros: Moscú. Documentaire. [http://bit.ly/2F62iu7; 20/02/2018].

Españoles en el mundo: Moscú. Émission. [http://bit.ly/2ESZSeU ; 21/02/2018].

GOFFMAN, Erving (1972) : Interaction Ritual, Essays to Face-to-Face Behavior, Harmondsworth : Penguin.

HALL, Edward (1973): La dimensión oculta. Enfoque antropológico del uso del espacio, (trad. de J. Hernández Orozco). Madrid: Instituto de Estudios de Administración Local, (Nuevo Urbanismo).

(1981): Beyond Culture. New York : Anchor Books.

(1989): El lenguaje silencioso, (trad. de C. Córdoba). Madrid: Alianza Editorial.

JOHNSON, Harold ; EKMAN, Paul ; FRIESEN, Wallace (1975) : "Communicative

Body Movements - American Emblems », (Danesi, M.). Semiotica. Berlin: De Gruyter, 15(4), 335-353.

KERBRAT-ORECCHIONI, Catherine (1992) : Les interactions verbales. T. II. Paris : Armand Colin.

(2001) : Les actes de langage dans le discours. Théorie et fonctionnement. Paris : Nathan.

(2011) : « Politesse, impolitesse, «non-politesse», «polirudesse» : aperçus théoriques et application aux débats politiques télévisuels », (Held, G., Helfrich, U.). Cortesia - Politesse - Cortesía. La cortesia verbal nella prospettiva romanistica. La politesse verbale dans une perspective romaniste. La cortesía verbal desde la perspectiva romanística. Berne : Peter Lang, 93-116. 
KREJDLIN, Grigorij (2008) : « Le langage du corps et la gestuelle (kinésique) comme champs de la sémiotique non-verbale : idées et résultats ». Cahiers slaves, $\mathrm{N}^{\circ} 9$ : 1-23, (trad. du russe de F. Daucé). [http://bit.ly/2CjkCPn ; 7/11/2017].

LAPIERRE, Dominique (2006): Érase una vez la URSS, (trad. de C. de Celis). Barcelona: Editorial Planeta.

PEASE, Allan \& Barbara (2006): El lenguaje del cuerpo, (trad. de I. Murillo). Barcelona: Amat.

RODRÍGUEZ, Belén (2009): "Priviet, San Petersburg". Blog Españoles en el mundo. [http://bit.ly/2EzNn7I; 20/02/2018].

SALVADOR, Carlos (2010): “Por qué esta gente no sonríe?” Blog RTVE. [http://bit. ly/2BThKaR; 27/02/2018].

STERNIN, Iossif (2013) : " 10 raisons pour lesquelles les Russes ne sourient pas ». Russia Beyond. [http://bit.ly/2CLfBuI ; 27/02/2018].

БЕРКОВА А.В., БЕРКОВА О.В., БЕРКОВ В.П., Как мы живем. Пособие по страноведению для изучающих русский язык, Изд. 2-е. СанктПетербург: Златоуст, 2003 [BERKOV, Valeriy et al. (2003): Comment nous vivons. Recommandations pratiques pour les apprenants de langue russe, $2 \mathrm{e}$ édition. Saint-Pétersbourg: Zlatoust].

ВОЛЬСКАЯ, Н. и др., Можно? Нельзя?: Практический минимум по культурной адаптации в русской среде. Москва: Русский язык. Курсы, 2001 [VOLSKAYA, Natalia et al. (2001): On peut? On ne peut pas? Le minimum pratique sur l'adaptation culturelle dans l'entourage russe. Moscou : Russki yazik].

ЕГОРОВА, Т.Н., Андрей Миронов и Я. Москва: Захаров, 2003 [YEGOROVA, Tatiana (2003) : Andreï Mironov et moi. Moscou : Zakharov].

Ольга, «Десять нелепых видов аватарок для Вконтакте», [Olga, « Les dix types d'avatars absurdes pour Vkontakte ", (22/07/2010)]. [http://bit.ly/2Do8nPh ; 9/02/2018].

ПЕРЕВОЗНИКОВА, А., Россия: страна и люди. Лингвострановедение: учебное пособие для изучающих русский язык как иностранный. Москва : Русский язык. Курсы, 2006 [PEREVOZNIKOVA, Alevtina (2006) : La Russie : le pays et les gens. Civilisation du pays : manuel didactique pour les étudiants de russe comme langue étrangère. Moscou : Russki yazik, Kursi].

«Почему европейцы чаще улыбаются, что у нас настолько больше проблем?» [Forum « Pourquoi les européens sourient-ils plus souvent ? Est-ce que nous avons beaucoup plus de problèmes? »]. [http://bit.ly/2CL7MFm; 27/02/2018].

«Почему русские, в отличие от европейцев или американцев, мало улыбаются?» [Forum " Pourquoi les Russes, à la différence des Européens et des Américains, sourient-ils peu ?»]. [http://bit.ly/2GNnRg6;27/02/2018].

«Почемуу нас люди, работающие в сфере обслуживания, наработе не улыбаются?» [Forum « Pourquoi nos gens qui travaillent dans le secteur tertiaire ne sourient-ils pas à leur poste ?»]. [http://bit.ly/2t0kAbp; 27/02/2018]. 
САНДАКОВА Е., «Развитие коммуникативной компетенции у студентов на основе изучения культурных жестов (на примере русского, фрранцузского и испанского языков)» // Роль иностранного языка в модернизации современного образовательного процесса: материалы Международной научно-практической конференции. 2 июня 2011 г., Барнаул / отв. ред., сост. Т.В. Скубневская. - Барнаул: Изд-во «Концепт», 2011. - C. 41-45. [SANDAKOVA, Elena (2011) : « Le développement de la compétence de communication chez les étudiants à l'aide de l'appréhension des gestes culturels (sur l'exemple du russe, du français et de l'espagnol) », (Skubnevskaia, T.V.). Le rôle de la langue étrangère dans la modernisation du processus éducatif actuel : matériaux de la conférence scientifique et pratique internationale, 2 juin 2011, Barnaoul. Barnaoul : Kontsept, 41-45].

СЕРГЕЕВА, А., Русские: стереотипы поведения, традиции, ментальность. Москва: Флинта, 2017 [SERGUEYEVA, Alla (2017) : Les Russes : stéreótypes de comportement, tradictions, mentalité. Moscou : Flinta].

\section{PROFIL ACADÉMIQUE ET PROFESSIONNEL}

Elena Sandakova est professeure de français à la chaire de Philologie française de l'Université d'Alicante depuis 2011. Ses matières enseignées à souligner sont Pragmatique française, Lexicologie française, Communication écrite en langue française, Langue française, Le français pour le tourisme et Apprentissage et enseignement du FLE (Master). Maîtrise en Philologie Française et Anglaise. Docteur en Linguistique Française. Thèse de Doctorat qualifiée cum laude dans le cadre du programme "Langues, cultures et leurs traductions dans l'espace méditerranéen » (Université d'Alicante), soutenue en 2016, sous la direction de Marina Aragón Cobo.

Recherche : sociolinguistique, langue-culture, sémiotique, pragmatique

Langues : russe (maternelle), français, espagnol, anglais, tatar, kazakh.

Fecha recepción del artículo: 15-11-2017

Fecha aceptación del artículo: 4-4-2017 